\title{
NAFLD e Ingesta de Fructosa en Altas concentraciones. Una Revisión de la Literatura
}

\author{
NAFLD and High Fructose Intake. A Review of Literature
}

\author{
Pamela Carvallo ${ }^{1,2}$; Eugenia Carvallo ${ }^{1,3}$; Sandra Barbosa-da-Silva ${ }^{4}$; \\ Carlos Alberto Mandarim-de-Lacerda ${ }^{4} \&$ Mariano del Sol ${ }^{1,5}$
}

CARVAllo, P.; CARVAllo, E.; BARBOSA-DA-SILVA, S.; MANDARIM-DE-LACERDA, C. A. \& DEL SOL, M. NAFLD e ingesta de fructosa en altas concentraciones. Una revisión de la literatura. Int. J. Morphol., 35(2):676-683, 2017.

RESUMEN Uno de los endulzantes más comúnmente utilizado es la fructosa. La fructosa es directamente metabolizada en el hígado y se puede transformar en glucosa, posteriormente es almacenada como glicógeno constituyéndose en una fuente de energía para los hepatocitos. Todo el exceso de fructosa se convierte en lípidos ejerciendo un efecto tóxico sobre el hígado, similar al producido por el exceso de alcohol, pudiendo provocar hígado graso no alcohólico (NAFLD). El objetivo de esta revisión es reunir hallazgos recientes en relación al efecto de la ingesta de fructosa en altas concentraciones y su relación con el NAFLD.

PALABRAS CLAVE: Hígado graso no alcohólico; esteatohepatitis no alcohólica; fibrosis hepática; Fructosa.

\section{INTRODUCCIÓN}

La enfermedad hepática por hígado graso no alcohólico (NAFLD) es consecuencia de múltiples factores de riesgo como sobrepeso, obesidad central, dislipidemia, resistencia a la insulina y Diabetes Mellitus tipo 2; todos ellos asociados con el síndrome metabólico (Adams et al., 2009). La evolución del NAFLD puede producir esteatohepatitis no alcohólica (NASH), fibrosis hepática y puede predisponer al desarrollo de hepatocarcinoma. El NAFLD se caracteriza por exceso de deposición de lípidos neutros (hepatoesteatosis) en el hígado, más del $5 \%$ de los hepatocitos están almacenando lípidos neutros (ésteres de triglicéridos y colesterol), el depósito de triglicéridos en el hígado es el resultado de un desequilibrio entre la cantidad de energía ingerida y la cantidad usada. El NAFLD comprende un espectro de enfermedad que va desde la estatosis (NAFL) a la esteatohepatitis no alcohólica (NASH) y la fibrosis (Bettermann et al., 2014; Petaja \& Yki-Jarvinen, 2016).

La ingesta excesiva de grasas y carbohidratos constituye uno de los factores de riesgo de presentar NAFLD. Uno de los endulzantes más comúnmente utilizados en la dieta occidental es la fructosa que es directamente metabolizada en el hígado y se transforma en glucosa, posteriormente es almacenada como glicógeno constituyéndose en una fuente de energía para los hepatocitos. Todo el exceso de fructosa se convierte en lípidos ejerciendo un efecto tóxico sobre el hígado, similar al producido por el exceso de alcohol, pudiendo provocar NAFLD (Lustig, 2010). Por tales razones los médicos alientan a sus pacientes a modificar la alimentación, reduciendo el consumo de grasa y carbohidratos simples y a perder el exceso de peso corporal con el fin de prevenir el daño hepático (Lytle \& Jump, 2016; Zheng et al., 2016).

Actualmente la alta prevalencia de obesidad y Diabetes Mellitus tipo 2 a nivel mundial ha provocado que el NAFLD sea considerado una entidad clínica relevante, constituyendo la principal causa de enfermedad hepática crónica e indicación de trasplante hepático en países desarrollados (Matherly \& Puri, 2012).

Epidemiología. El NAFLD afecta al $33 \%$ de la población occidental, constituyendo un importante problema de salud pública. Se presenta en el 20-30\% de personas no obesas y

\footnotetext{
${ }^{1}$ Doctorado en Ciencias Morfológicas, Universidad de La Frontera, Temuco, Chile.

${ }^{2}$ Universidad Católica de Temuco, Chile.

${ }^{3}$ Universidad Mayor, Sede Temuco, Chile.

${ }^{4}$ Universidade do Estado de Rio de Janeiro, Brasil.

${ }^{5}$ Centro de Excelencia en Ciencias Morfológicas y Quirúrgicas, Universidad de La Frontera, Temuco, Chile.

Proyecto DIUFRO-FAPERJ No FPJ15-0013.
} 
sobre el $75 \%$ en pacientes obesos (Jahn et al., 2016). La prevalencia de NAFLD es cada vez más alta, existen diferencias étnicas siendo mayor su frecuencia en hispanos que en caucásicos y afroamericanos (Chalasani et al., 2012). Un estudio realizado por la Escuela de Medicina de la Pontificia Universidad Católica de Chile, reveló que $23 \%$ de los sujetos eran portadores de NAFLD. La resistencia a la insulina y obesidad fueron factores asociados a la presencia de esta condición (Riquelme et al., 2009). La encuesta nacional de salud 2009-2010 indicó que del total de la población estudiada $64,5 \%$ presentaba exceso de peso, $27 \%$ HTA, $31 \%$ hipertrigliceridemia, 9,4\% Diabetes Mellitus tipo 2 y $35 \%$ síndrome metabólico.

Diagnóstico de NAFLD y fibrosis. Los métodos más utilizados para el diagnóstico de NAFLD y NASH en estudios clínicos son: ecografía abdominal, espectroscopía por resonancia magnética, medición de aminotranferasas y biopsia hepática (Bugianesi et al., 2002).

Evaluación no invasiva de la necroinflamación: La NAFLD puede provocar incremento leve, crónica y asintomática de aminotransferasas. La alanino aminotransferasa (ALT), es una enzima específica de origen hepático y ha sido utilizada como examen de pesquisa por ser de bajo costo, confiable y ampliamente disponible (Kim et al., 2008). Sin embargo, existen estudios que han demostrado que aproximadamente el $80 \%$ de los individuos con hígado graso tiene niveles de ALT dentro de rango normal, a raíz de lo cual no sería un indicador sensible de NAFLD (Adams \& Angulo, 2007). Se considera como score predictivo al NASH, que incluye las variables: edad, sexo, talla, peso, alfa-macroglobulina, hepatoglobina, apolipoproteina A1, bilirrubina total, GGT, ALT, AST, triglicéridos, colesterol y glicemia (Tanwar et al., 2013).

La ecotomografía abdominal es el método de imagenología más empleado por ser no invasivo, ampliamente disponible y de bajo costo (Hashimoto et al., 2013). La sensibilidad en la detección de esteatosis comparado con la histológica varía entre 82-94 \% y su especificidad sería sobre $82 \%$ (Mishra \& Younossi, 2007). Existen otras técnicas como la tomografía computarizada, que no es más precisa que la ecografía para detectar esteatosis y tiene la desventaja de su mayor costo y el uso de radiación. Por otro lado, la resonancia magnética, si bien puede ser más sensible que la ecografía para el diagnóstico de esteatosis leve, consume más tiempo y es de mayor costo (Machado \& Cortez-Pinto, 2014).

Evaluación no invasiva de la fibrosis: Estos métodos se basan en la determinación del grado de rigidez del hígado por elastografía transitoria (Fibroscan), ARFI(Acoustic
Radiation Force Impulse) elastografía en tiempo real y elastografía por resonancia (Yoshioka et al., 2015).

Biopsia hepática: es el único método validado para diferenciar NAFLD de EHNA, una vez que se ha descartado el consumo de alcohol. Evalúa el grado de esteatosis, inflamación e injuria hepatocitaria así como el de fibrosis (Chalasani et al., 2012; Singh et al., 2015). Los análisis histológicos más utilizados para el estudio de daño hepático son: score de Brunt (Brunt et al., 1999; Brunt, 2000) y el de NAS (NAFLD Activity Score) desarrollado por el NASH Clinical Research Network (Kleiner et al., 2005). Sin embargo, el método de "scores" para evaluar el daño hepático es subjetivo y requiere una amplia formación del patólogo, por lo que es poco reproducible. Para superar esta dificultad se han desarrollado procedimientos con estereología y análisis de imágenes (Aguila et al., 2003). Recientemente, los cortes de hígado teñidos con hematoxilina y eosina (H-E) fueron comparados con secciones congeladas teñidas con Oil Red-O (ORO). Además, la densidad de volumen de la esteatosis ( $\mathrm{Vv}$ [esteatosis, hígado]) se midió por conteo de puntos (P-C, secciones $\mathrm{H}-\mathrm{E} \mathrm{u}$ ORO) o por análisis de imagen (I-A, secciones ORO). La correlacion entre $\mathrm{Vv}$ [esteatosis, hígado] y los niveles de triglicéridos hepáticos (HT) fue grande y significativa en todos los métodos. Por lo tanto, los métodos eran apropiados y reproducibles. En P-C y H-E, existe una ligera sobrestimación de esteatosis en los animales en comparación con secciones congeladas y ORO. En las secciones congeladas, las diferencias entre P-C e I-A no son significativas (Catta-Preta et al., 2011). Muy recientemente se utilizó estereología en las biopsias de hígado con resultados muy alentadores (St Pierre et al., 2016), incluso teniendo en consideración que la biopsia hepática es un procedimiento invasivo y solo representa el 1:50.000 de todo el volumen hepático (Sumida et al., 2014).

\section{a) Patogénesis}

Factores de riesgo: El síndrome metabólico es un fuerte predictor de la presencia de NASH en pacientes con NAFLD. Así mismo, individuos mayores a 50 años, la obesidad visceral, la hipertensión arterial, la Diabetes Mellitus tipo 2, el índice AST/ALT>1 (AST: aspartato aminotransferasa; ALT: alanino aminotransferasa) y la trombocitopenia también se constituyen en factores de riesgo de NASH y fibrosis avanzada (Machado \& Cortez-Pinto, 2006; Chalasani et al., 2012; Berlanga et al., 2014).

La NASH se relaciona con dislipidemia y resistencia a la insulina (RI). Esta última se produce por la infiltración de macrófagos en el tejido adiposo visceral, en donde se desencadena una respuesta inflamatoria, con secreción de adipoquinas con efectos pro-inflamatorios y pro-fibróticos. Este fenómeno se potencia en el hígado de tal manera que, 
tanto por vía sistémica, como intrahepática existiría un exceso de citoquinas pro-inflamatorias, tales como factor de necrosis tumoral-alfa (TNF-a) e interleuquina 6 (IL-6) (Attie \& Scherer, 2009; Fotbolcu \& Zorlu, 2016). Múltiples factores estarían implicados en el proceso inicial de la inflamación en el tejido adiposo, que incluyen isquemia relativa y la producción de hipoxia inducida por factor-1, microflora intestinal selectiva, respuesta inflamatoria mediada por microflora y hormonas como la leptina. Una consecuencia importante de la RI es el aumento de la actividad lipolítica y liberación de ácidos grasos libres (AGL) a la circulación, de donde son captados por el hepatocito e inducen lipotoxicidad. La RI también permite potenciar la sensibilidad a la insulina en su efecto lipogénico en el hígado (Matherly \& Puri).

Otros sistemas en juego incluyen a los canabinoides, los que han demostrado ser un importante determinante de la lipogénesis de novo hepático (Gruen et al., 2007; Nguyen \& Sanyal, 2012). La diferencia entre esteatosis y esteatohepatitis, radica en el mayor grado de apoptosis e inflamación asociados a balonamiento celular. La NASH presenta mayor propensión al desarrollo de cirrosis. Diversos mecanismos estarían implicados en la injuria celular que incluye lipotoxicidad por AGL, stress oxidativo (SO), stress de retículo endoplasmático (RE) y activación del sistema inmune y de citoquinas, todas ellas mediadas por AGL que se traducen en daño celular. Existen diferentes fuentes de SO, la NASH se asocia a daño mitocondrial y alteración en el trasporte de electrones; activación del citocromo P450 2 capaz de generar oxígeno reactivo; también la disfunción del peroxisoma. EL SO activa vías inflamatorias como cJun N-terminal (una quinasa) y factor nuclear kappa B. El glutation es el principal antioxidante y su recambio aumenta en condiciones de SO. La apoptosis ocurre tanto por lipotoxicidad como por SO. El stress del RE contribuye a la apoptosis. Finalmente, la progresión de la enfermedad es consecuencia del desbalance entre injuria, reparación y fibrosis (Nguyen \& Sanyal, 2012; Schultz et al., 2015).

Factores genéticos: Estudios genéticos han determinado que la heredabilidad del esteatosis hepática es de aproximadamente un 39 \% (Schwimmer et al., 2009). Los hombres desarrollan NASH con mayor frecuencia que las mujeres hasta los 60 años y luego esta tendencia se revierte (Browning et al., 2004). La mutación I148M en el gen PNPLA3 (patatinlike phospholipase domain-contining 3) se ha asociado con el desarrollo de NAFLD (Huang et al., 2010). Esto puede explicar las diferencias en la susceptibilidad al NAFLD entre diferentes etnias. La proteína PNPLA3 es inducida por la insulina a través de vías controladas por LXR y SREBP1c, algunas investigaciones sugieren que la mutación I148M provoca disminución de la actividad de la hidrolasa TG y desarrolla esteatosis, existen estudios que han determinado que otras regiones genéticas confieren riesgo de desarrollar NASH incluyendo NCAN, PPP1R3B y en asiáticos la apolipoproteina C3 (He et al., 2010).

\section{Factores de la dieta:}

Dieta alta en grasas: Los hábitos alimentarios pueden afectar la incidencia de esteatosis hepática, una dieta alta en grasas aumenta la actividad de endocanabinoides, mediadores lipídicos que actúan sobre los receptores canabinoides para alterar el metabolismo de los lípidos. Los receptores canabinoides 1 (CB1) tienen efectos sobre el tejido adiposo y hepático (Osei-Hyiaman et al., 2005; Kunos \& OseiHyiaman, 2008), su estimulación aumenta la liberación de ácidos grasos desde el tejido adiposo disminuyendo los niveles de adiponectina, aumentando la RI y disminuyendo la beta-oxidación de ácidos grasos (Tam et al., 2011).

- Dieta alta en carbohidratos: Una dieta alta en carbohidratos simples también puede provocar daño hepático. Los endulzantes más utilizados en la actualidad son azúcares como: sacarosa, fructosa y glucosa, la ingesta de fructosa en altas concentraciones es otra posible causa de esteatosis hepática. La fructosa y glucosa son monosacáridos presentes en pequeñas cantidades en frutas y miel, mientras la sacarosa es un disacárido formado por una molécula de glucosa unida a una molécula de fructosa a través de alfa-14 puentes de glicosido y se encuentra en grandes cantidades en la caña de azúcar y remolacha (Tappy \& Le, 2010, 2015). El consumo de fructosa ha aumentado a nivel mundial en los últimos años, especialmente en países occidentales.

Dieta alta en fructosa: El azúcar de mesa está compuesta en partes iguales por glucosa y fructosa, que es dos veces más dulce. El jarabe de fructosa, está constituido por $45 \%$ de glucosa y $55 \%$ de fructosa, mientras la glucosa puede ser metabolizada por cualquier órgano, la fructosa solo es metabolizada por el hígado. El consumo del azúcar y el jarabe de maíz dañan el hígado, especialmente si está incluida en bebidas o jugos pues se metabolizan más rápido y producen mayor grado de obesidad (Mamikutty et al., 2014). Mientras más alto es el consumo de fructosa se genera mayor aumento del tamaño y almacenamiento de glicógeno en el hígado, así como alteración de la expresión de FAS (ácido graso sintasa) y SCD-1 (steroyl-CaA desnaturasa-1) y elevación de ácidos grasos (Kanuri \& Bergheim, 2013; Maslak et al., 2015). Por otra parte, las dietas que combinan alto consumo de grasa y fructosa producen mayor aumento de la inflamación y daño metabólico (Lee et al., 2015; Ferrere et al., 2016). Un estudio realizado en ratones C57/BL6J con dieta alta en fructosa (60 \% del total de calorías) y alta en etanol por 18 semanas determinó que la fructosa potencia el daño hepático producido por alcohol (Song et al., 2016). 
a) Efectos de la Fructosa: $\mathrm{Si}$ se consume fructosa en altas cantidades el hígado la transforma en grasa lo que provoca resistencia a la insulina. Cuando las células se hacen resistentes a la insulina, el páncreas intenta regular los niveles de glicemia produciendo más insulina provocando mayor acumulación de tejido adiposo. También bloquea la acción de la leptina provocando sensación de hambre. Los niveles altos de insulina aumentan la presión arterial y disminuyen el HDL, provocando síndrome metabólico, obesidad y NAFLD. Es decir, el azúcar provoca en el hígado el mismo daño que el consumo de alcohol (Lustig, 2013). Cuando la fructosa es consumida en cantidades moderadas ya sea como jarabe de maíz o sacarosa no se almacena tejido adiposo ectópico en hígado o músculo esquelético (Bravo et al., 2013; Heden et al., 2014). La ingesta de fructosa acelera las alteraciones metabólicas asociadas con el envejecimiento tales como la resistencia a la insulina e intolerancia a la glucosa (Lozano et al., 2016). La obesidad puede intensificar la elevación de la RI en adolescentes entre 12-16 años (Lin et al., 2016).

De acuerdo a los estudios de Pektas et al., 2015, la ingesta de fructosa por largo tiempo puede afectar la función metabólica de manera diferente en cada sexo, provocando aumento del peso corporal de ratas machos, pero no de hembras. También, se observó que Lipocalin-2 (LCN-2) una proteína que inhibe la actividad macrofágica, aumentó en hígado y suero asociada con inflamación hepática y alteración de la función mitocondrial. Los indicadores de estrés oxidativo y apoptosis de los hepatocitos fueron elevados y la expresión de LCN-2 se localizó en granulocitos hepáticos mediante inmunohistoquímica. Los niveles séricos de HDL-colesterol, LCN-2, leptina y triglicéridos fueron elevados (Alwahsh et al., 2014). Otros autores determinaron que la fructosa produce moderada esteatosis macrovacuolar y necroinflamación, con ausencia de fibrosis periportal y niveles significativamente más altos de triglicéridos, ALT y adiponectina así como TNF-a, glucosa e insulina (FakhourySayegh et al., 2015; Liu et al., 2015).

Según Rodrigues et al. (2016) el cosumo elevado de fructosa disminuye la actividad de las lipasas citosólicas en el hígado y tejido adiposo y aumenta la lipogénesis. Histopatológicamente la fructosa produce aumento en el volumen de las gotas de lípidos en hepatocitos y en niveles séricos de Triacilglicerol (TAG), pero reduce los niveles de insulina postprandial

De acuerdo a los estudios de Dowman et al. (2014), realizados en ratones, la expresión de acetyl-CoA carbolxilasa 1 y FAS, genes claves en la regulación de la lipogénesis, aumentaron así como la expresión de carnitina palmitoil transferasa (CPT-1), enzima necesaria para la beta- oxidación de ácidos grasos de cadena larga. La dieta alta en fructosa no afectó el peso corporal de manera significativa (Tillman et al., 2014; Sharma et al., 2015).

Un estudio realizado en ratones C57/BL6 demostró que IRE1- mediado por la activación de JNK, más que la acumulación de lípidos, es el gatillante para establecer la resistencia a la insulina (RI) en el hígado inducida por una dieta alta en fructosa (35\%), sin embargo, esto no excluye la posibilidad de que la acumulación de lípidos por de novo lipogénesis (DNL) pueda contribuir al desarrollo de la RI hepática a largo plazo (Sun et al., 2015). Por otra parte, Jarukamjorn et al. (2016) señalaron que la actividad de enzimas SOD (superóxido dismutasa), CAT (catalasa) y GPx (Glutation peroxidasa) aumentaba mientras que el almacenamiento de GSH (Glutation reducido) disminuía, determinando que el desequilibrio en la antioxidación aumenta el riesgo y la progresión de NAFLD.

En un estudio reciente, nos hemos planteado investigar el rol de la metformina en un modelo animal de lesión hepática causada por la ingesta de fructosa, centrándose en los marcadores moleculares de la lipogénesis, la beta-oxidación y las defensas antioxidantes. Ratones machos C57BL6 de tres meses de edad se dividieron en grupo control (C) y grupo fructosa (F, $47 \%$ de fructosa), mantenidos durante diez semanas. Posteriormente, los grupos recibieron durante otras ocho semanas: control (C), control + Metformina (CM), fructosa (F) y fructosa + Metformina (FM). El grupo fructosa desarrolló esteatosis hepática, resistencia a la insulina y menor sensibilidad a la insulina en asociación con mayores niveles de RNAm de proteínas ligadas a la lipogénesis de novo y aumento de la peroxidación lipídica. La fructosa disminuyó la expresión de mRNA de las enzimas antioxidantes, y de las proteínas responsables de la biogénesis mitocondrial. La metformina redujo la lipogénesis de novo y aumentó la expresión de proteínas relacionadas con la biogénesis mitocondrial, aumentando así la beta-oxidación y disminuyendo la peroxidación lipídica. Además, la metformina reguló positivamente la expresión y la actividad de las enzimas antioxidantes, proporcionando una defensa contra el aumento de la generación de especies reactivas de oxígeno. Por lo tanto, una reducción significativa en la acumulación de triglicéridos en el hígado, esteatosis y peroxidación de lípidos se observó en el grupo FM. En conclusión, la fructosa aumenta la lipogénesis de novo, reduce las defensas antioxidantes y disminuye la biogénesis mitocondrial. Después de un período prolongado de consumo de fructosa, el tratamiento con metformina, incluso en la continuación de la ingesta de fructosa, puede revertir, al menos parcialmente, la lesión hepática y previene la progresión de NAFLD a un estado más severo (Karise et al., 2017). 
b) Metabolismo de la Fructosa: Los mecanismos por los cuales la fructosa es metabolizada difieren de la glucosa. Después de que la fructosa es absorbida desde el intestino, es metabolizada en el hígado directamente, siendo independiente de la insulina. En el hígado, la fructosa tiene dos destinos: transformarse en glucosa y ser almacenada como glicógeno o ser usada como una fuente de energía para los hepatocitos. Así, excepto cuando el almacenamiento de glicógeno es bajo, todo el exceso de fructosa será convertido en tejido adiposo, preferentemente metabolizado en lípidos, contrariamente a lo que sucede con la glucosa (Lustig, 2010).

Algunos estudios sugieren que el daño hepático producido por la fructosa se origina del rápido metabolismo de la fructosa catalizado por fructoquinasa C (Stanhope et al., 2013), la cual genera el sustrato para el desarrollo DNL en el hígado y produce aumento de los niveles de ácido úrico. Por otra parte, DiNicolantonio et al. (2015) señalaron que la fructosa aumenta la liberación de ácidos grasos libres y de VLDL produciendo acumulación de lípidos intramuscular y resistencia a la insulina en el músculo esquelético. La disminución celular de ATP produce reducción de unión celular a la insulina y el número de receptores de insulina. El aumento de la inflamación y estrés oxidativo produce daño de las células b del páncreas y disminuye la secreción de insulina.

Stanhope (2016) señaló que el aumento de lípidos en el hígado promueve la producción y secreción de VLDL, provocando una elevación de los niveles de TG y colesterol LDL (dislipidemia), aumentado el riesgo CVD. Según este autor, también puede producir RI hepática por aumento de los niveles de DAG (diacilglicerol) el cual activa la novel proteinquinasa $\mathrm{C}(\mathrm{nPKC})$ y produce fosforilacion serina (serine $\mathrm{P}$ ) de los receptores de insulina y receptores de insulina sustrato 1 (IRS-1) impidiendo la acción de la insulina. Debido a la RI selectiva, DNL es más fuertemente activado en la RI hepática, provocando un círculo vicioso. La RI hepática también aumentaría la producción y secreción de VLDL por aumento de la disponibilidad de apolipoproteina B (apo B) y síntesis de ApoC3 y aumentando la expresión de la proteína de transferencia triglicérido microsomal (MTP). Esto exacerba y sostiene la exposición a TG circulantes aumentando la acumulación de lípidos en el músculo, impidiendo la señalización de la insulina y produciendo RI en todo el cuerpo.

La fosforilación de la fructosa catalizada por la fructoquinasa a fructosa 1-fosfato, (resultado de la conversión de ATP a AMP y a una disminución del fosfato inorgánico), según Stanhope et al. (2015) conduce a la producción de ácido úrico por la vía de la degradación de la purina. Así, altos niveles de ácido úrico estarían asociados a NAFLD, enfermedad cardiovascular y síndrome metabólico. La exposición a la fructosa en el intestino e hígado y el aumento de tejido adiposo visceral producido por la fructosa puede provocar respuestas inflamatorias que posteriormente promueven la acumulación de lípidos en el hígado y/o impiden la señalización de la insulina hepática.

El metabolismo de la fructosa favorece DNL con mayor intensidad que el consumo de dietas altas en grasas y la DNL hepática corresponde a la alteración central en NAFLD. Por tanto, una alteración del metabolismo de la fructosa en el hígado podría proporcionar una nueva opción terapéutica para el NAFDL. La exposición crónica a fructosa ejerce síntomas de tolerancia y abstinencia similares al abuso crónico de etanol, siendo también una sustancia adictiva. La única diferencia entre el etanol y la fructosa es que esta no se metaboliza en el sistema nervioso central y no ejerce un efecto depresivo sobre las neuronas (Lustig, 2013; Schultz et al., 2013).

c) Efectos de la fructosa sobre las células estrelladas hepáticas (HSC): Dowman et al. reportaron que el desarrollo de un modelo de NASH en ratones C57/BL6 replica muchas de las características vistas en humanos, así como la activación de células estrelladas hepáticas (HSC) en respuesta a cambios en la dieta y estilos de vida. Este estudio demostró que una dieta alta en grasa y fructosa y el sedentarismo pueden inducir NASH y hepatocarcinogénesis en un plazo de seis y doce meses, respectivamente. Las células estrelladas hepáticas (HSC), las principales células fibrogénicas del hígado, sufren apoptosis después del cese del daño hepático, contribuyendo de este modo a la resolución de la fibrosis hepática. Sin embargo, según Giraudi et al. (2015) la proximidad entre hepatocitos y células estrelladas es necesaria para la iniciación del proceso fibrótico.

En un estudio realizado por Troeger et al. (2012) en ratones, determinaron que la reversión de la activación de HSC contribuye a la finalización de la fibrogénesis ya que esta desactivación sirve como estímulo regenerativo aumentando la apoptosis de los miofibroblastos. Por otra parte, la Rosuvastatina, una estatina más reciente, mitiga la esteatosis hepática modulando el balance PPAR, favoreciendo PPARalfa sobre PPAR-gamma. Los efectos beneficiosos de Rosuvastatina se acompañan por una disminución de la resistencia a la insulina, el perfil anti-inflamatorio adipokina, y la activación de HSC, evitando la progresión de NAFLD y la aparición de NASH (Marinho et al., 2016).

d) Efectos de la reducción de la ingesta de fructosa: Los hallazgos imagenológicos han demostrado que la reducción de la ingesta de fructosa mejora la esteatosis hepática, me- 
jora la sensibilidad a la insulina y la inflamación hepática. Los niveles de AST, ALT, TG, colesterol total, glicemia e insulina se reducen, siendo similares a los del grupo control, luego de suspender la ingesta de fructosa por 4 semanas, por tanto, la reducción de la ingesta de fructosa en la dieta a 5\% del total de calorías (nivel sugerido por la OMS) se ha demostrado que mejora la tolerancia a la glucosa en humanos y disminuye la prevalencia de diabetes y alteraciones metabólicas que a menudo la preceden (DiNicolantonio et al.).

La limitación de alimentos y bebidas que contienen azúcar adicionada, particularmente fructosa, puede ser una de las más efectivas estrategias para evitar el daño hepático (DiNicolantonio et al.), pudiendo generar una regresión histológica (Malhotra \& Beaton, 2015).

De acuerdo a los estudios de Dowman et al., la restauración de la actividad de PPAR-a recupera la función metabólica de las mitocondrias y RE, alivia la hipertrigliceridemia sistémica y mejora la esteatosis hepática. La activación de la HCS es regulada por citoquinas y ROS liberadas por los hepatocitos dañados. Por lo tanto, la supresión del estrés oxidativo y la inhibición de la activación de las HCS podría proporcionar una alternativa terapéutica en el tratamiento de la fibrosis hepática (Ramachandran et al., 2015; Sodhi et al., 2015).

Stanhope et al. (2013) y Bettermann et al. (2014), indicaron que la reducción del consumo de fructosa o el reemplazo de bebidas azucaradas por bebidas endulzadas artificialmente tienen efectos beneficiosos sobre los factores de riesgo de enfermedades metabólicas o sobre el índice de masa corporal (IMC) en niños.

Finalmente, para Kolderup \& Svihus (2015) y Malhotra \& Beaton (2015) la pérdida del 7-10 \% del peso corporal, la limitación de la ingesta de altas concentraciones de fructosa y alimentos altos en grasa y glucosa, combinadas con actividad física regular e intensa podrían revertir el NAFLD y la esteatosis hepática.

\section{CONCLUSIÓN}

El NAFLD es una enfermedad frecuente en pacientes obesos y/o con síndrome metabólico y tiene una alta prevalencia a nivel mundial y nacional. Para poder prevenir o tratar esta patología será necesario conocer más sobre su patogénesis. Una de las principales causas es sin lugar a dudas, el alto consumo de lípidos y carbohidratos simples como la fructosa. De esta manera, conocer los mecanismos que causan estas las alteraciones metabólicas se podrán elaborar mejoras en las actuales estrategias terapéuticas.

Lo más importante es diagnosticar el NAFLD de forma precoz para controlar lo factores de riesgo y evitar la progresión de la enfermedad. Las principales medidas terapéuticas y de prevención son las modificaciones en la dieta, la disminución de peso y el ejercicio físico. Estas medidas permitirán mejorar la insulino-resistencia, los niveles de aminotransferasas, esteatosis hepática e esteatohepatititis.

CARVALLO, P.; CARVALLO, E.; BARBOSA-DA-SILVA, S.; MANDARIM-DE-LACERDA, C.A. \& DEL SOL, M. NAFLD and high fructose intake. A Review of Literature. Int. J. Morphol., 35(2):676-683, 2017.

SUMMARY: One of the most commonly used sweeteners is fructose. Fructose is directly metabolized in the liver and can be converted into glucose, later stored as glycogen constituting a source of energy for the hepatocytes. All excess fructose is converted into lipids by exerting a toxic effect on the liver, similar to that produced by excess of alcohol, and can cause nonalcoholic fatty liver (NAFLD). The aim of this review is to gather recent findings regarding the effect of fructose intake at high concentrations and its relationship with NAFLD.

KEY WORDS: Non-alcoholic fatty liver; Nonalcoholic steatohepatitis; Hepatic fibrosis; Fructose.

\section{REFERENCIAS BIBLIOGRÁFICAS}

Adams, L. A. \& Angulo, P. Role of liver biopsy and serum markers of liver fibrosis in non-alcoholic fatty liver disease. Clin. Liver Dis. 11(1):25-35, 2007.

Adams, L. A.; Waters, O. R.; Knuiman, M. W.; Elliott, R. R. \& Olynyk, J. K. NAFLD as a risk factor for the development of diabetes and the metabolic syndrome: an eleven-year follow-up study. Am. J. Gastroenterol., 104 (4):861-7, 2009.

Aguila, M. B.; Pinheiro, A. R.; Parente, L. B. \& Mandarim-de-Lacerda, C.A. Dietary effect of different high-fat diet on rat liver stereology. Liver Int., 23(5):363-70, 2003

Alwahsh, S. M.; Xu, M.; Schultze, F. C.; Wilting, J.; Mihm, S.; Raddatz, D. \& Ramadori, G. Combination of alcohol and fructose exacerbates metabolic imbalance in terms of hepatic damage, dyslipidemia, and insulin resistance in rats. PLoS One, 9(8):e104220, 2014.

Attie, A. D. \& Scherer, P. E. Adipocyte metabolism and obesity. J. Lipid Res., 50 Suppl. S395-9, 2009.

Berlanga, A.; Guiu-Jurado, E.; Porras, J. A. \& Auguet, T. Molecular pathways in non-alcoholic fatty liver disease. Clin. Exp. Gastroenterol., 7:221-39, 2014.

Bettermann, K.; Hohensee, T. \& Haybaeck, J. Steatosis and steatohepatitis: complex disorders. Int. J. Mol. Sci, 15(6):9924-44, 2014.

Bravo, S.; Lowndes, J.; Sinnett, S.; Yu, Z. \& Rippe, J. Consumption of sucrose and high-fructose corn syrup does not increase liver fat or ectopic fat deposition in muscles. Appl. Physiol. Nutr. Metab., 38(6):681-8, 2013.

Browning, J. D.; Szczepaniak, L.S.; Dobbins, R.; Nuremberg, P.; Horton, J. D.; Cohen, J. C.; Grundy, S. M. \& Hobbs, H. H. Prevalence of hepatic 
steatosis in an urban population in the United States: impact of ethnicity. Hepatology, 40(6):1387-95, 2004.

Brunt, E. M. Grading and staging the histopathological lesions of chronic hepatitis: the Knodell histology activity index and beyond. Hepatology, 31(1):241-6, 2000.

Brunt, E. M.; Janney, C. G.; Di Bisceglie, A. M.; Neuschwander-Tetri, B.A. \& Bacon, B.R. Nonalcoholic steatohepatitis: a proposal for grading and staging the histological lesions. Am. J. Gastroenterol., 94(9):2467-74, 1999.

Bugianesi, E.; Leone, N.; Vanni, E.; Marchesini, G.; Brunello, F.; Carucci, P.; Musso, A.; De Paolis, P.; Capussotti, L.; Salizzoni, M. \& Rizzetto, M. Expanding the natural history of nonalcoholic steatohepatitis: from cryptogenic cirrhosis to hepatocellular carcinoma. Gastroenterology, 123(1):134-40, 2002.

Catta-Preta, M.; Mendonca, L. S.; Fraulob-Aquino, J.; Aguila, M.B. \& Mandarim-de-Lacerda, C. A. A critical analysis of three quantitative methods of assessment of hepatic steatosis in liver biopsies. Virchows Arch., 459(5):477-85, 2011.

Chalasani, N.; Younossi, Z.; Lavine, J. E.; Diehl, A. M.; Brunt, E. M.; Cusi, K.; Charlton, M. \& Sanyal, A. J. The diagnosis and management of non-alcoholic fatty liver disease: practice Guideline by the American Association for the Study of Liver Diseases, American College of Gastroenterology, and the American Gastroenterological Association. Hepatology, 55(6):2005-23, 2012.

DiNicolantonio, J. J.; O'Keefe, J. H. \& Lucan, S. C. Added fructose: a principal driver of type 2 diabetes mellitus and its consequences. Mayo Clin. Proc., 90(3):372-81, 2015.

Dowman, J. K.; Hopkins, L. J.; Reynolds, G. M.; Nikolaou, N.; Armstrong, M. J.; Shaw, J. C.; Houlihan, D. D.; Lalor, P. F.; Tomlinson, J.W.; Hubscher, S. G. \& Newsome, P. N. Development of hepatocellular carcinoma in a murine model of nonalcoholic steatohepatitis induced by use of a high-fat/fructose diet and sedentary lifestyle. Am. J. Pathol., 184(5):1550-61, 2014.

Fakhoury-Sayegh, N.; Trak-Smayra, V.; Khazzaka, A.; Esseily, F.; Obeid, O.; Lahoud-Zouein, M. \& Younes, H. Characteristics of nonalcoholic fatty liver disease induced in wistar rats following four different diets. Nutr. Res. Pract., 9(4):350-7, 2015.

Ferrere, G.; Leroux, A.; Wrzosek, L.; Puchois, V.; Gaudin, F.; Ciocan, D.; Renoud, M. L.; Naveau, S.; Perlemuter, G. \& Cassard, A. M. Activation of Kupffer Cells Is Associated with a Specific Dysbiosis Induced by Fructose or High Fat Diet in Mice. PLoS One, 11(1):e0146177, 2016.

Fotbolcu, H. \& Zorlu, E. Nonalcoholic fatty liver disease as a multi-systemic disease. World J. Gastroenterol., 22(16):4079-90, 2016.

Giraudi, P. J.; Becerra, V. J.; Marin, V.; Chavez-Tapia, N. C.; Tiribelli, C. \& Rosso, $\mathrm{N}$. The importance of the interaction between hepatocyte and hepatic stellate cells in fibrogenesis induced by fatty accumulation. Exp. Mol. Pathol., 98(1):85-92, 2015.

Gruen, M. L.; Hao, M.; Piston, D. W. \& Hasty, A.H. Leptin requires canonical migratory signaling pathways for induction of monocyte and macrophage chemotaxis. Am. J. Physiol. Cell Physiol., 293(5):C14818, 2007.

Hashimoto, E.; Taniai, M. \& Tokushige, K. Characteristics and diagnosis of NAFLD/NASH. J. Gastroenterol. Hepatol., 28 Suppl. 4:64-70, 2013.

He, S.; McPhaul, C.; Li, J.Z.; Garuti, R.; Kinch, L.; Grishin, N.V.; Cohen, J.C. \& Hobbs, H.H. A sequence variation (I148M) in PNPLA3 associated with nonalcoholic fatty liver disease disrupts triglyceride hydrolysis. $J$. Biol. Chem., 285 (9):6706-15, 2010.

Heden, T. D.; Liu, Y.; Park, Y. M.; Nyhoff, L. M.; Winn, N. C. \& Kanaley, J.A. Moderate amounts of fructose- or glucose-sweetened beverages do not differentially alter metabolic health in male and female adolescents. Am. J. Clin. Nutr., 100(3):796-805, 2014.

Huang, Y.; He, S.; Li, J. Z.; Seo, Y. K.; Osborne, T. F.; Cohen, J. C. \& Hobbs, H. H. A feed-forward loop amplifies nutritional regulation of PNPLA3. Proc. Natl. Acad. Sci. USA, 107(17):7892-7, 2010.

Jahn, D.; Rau, M.; Wohlfahrt, J.; Hermanns, H. M. \& Geier, A. NonAlcoholic Steatohepatitis: From Pathophysiology to Novel Therapies. Dig. Dis., 34(4):356-63, 2016.
Jarukamjorn, K.; Jearapong, N.; Pimson, C. \& Chatuphonprasert, W. A High-Fat, High-Fructose Diet Induces Antioxidant Imbalance and Increases the Risk and Progression of Nonalcoholic Fatty Liver Disease in Mice. Scientifica, 2016:5029414, 2016.

Kanuri, G. \& Bergheim, I. In vitro and in vivo models of non-alcoholic fatty liver disease (NAFLD). Int. J. Mol. Sci., 14(6):11963-80, 2013.

Karise, I.; Ornellas, F.; da Silva, S. B.; Matsuura, C.; del Sol, M.; Aguila, M. B. \& Mandarim-de-Lacerda, C. A. Liver and Metformin: lessons of a fructose diet in mice. Biochimie Open, 4:19-30, 2017.

Kim, W. R.; Flamm, S. L.; Di Bisceglie, A. M.; Bodenheimer, H. C. \& Public Policy Committee of the American Association for the Study of Liver, D. Serum activity of alanine aminotransferase (ALT) as an indicator of health and disease. Hepatology, 47(4):1363-70, 2008.

Kleiner, D. E.; Brunt, E. M.; Van Natta, M.; Behling, C.; Contos, M. J.; Cummings, O. W.; Ferrell, L. D.; Liu, Y. C.; Torbenson, M. S.; UnalpArida, A.; Yeh, M.; McCullough, A. J.; Sanyal, A. J. \& Nonalcoholic Steatohepatitis Clinical Research, N. Design and validation of a histological scoring system for nonalcoholic fatty liver disease. Hepatology, 41(6):1313-21, 2005.

Kolderup, A. \& Svihus, B. Fructose Metabolism and Relation to Atherosclerosis, Type 2 Diabetes, and Obesity. J. Nutr. Metab., 2015: 823081, 2015.

Kunos, G. \& Osei-Hyiaman, D. Endocannabinoids and liver disease. IV. Endocannabinoid involvement in obesity and hepatic steatosis. Am. J. Physiol. Gastrointest. Liver Physiol., 294(5):G1101-4, 2008.

Lee, J. S.; Jun, D. W.; Kim, E. K.; Jeon, H. J.; Nam, H. H. \& Saeed, W. K. Histologic and Metabolic Derangement in High-Fat, High-Fructose, and Combination Diet Animal Models. ScientificWorld Journal, 2015: 306326, 2015.

Lin, W. T.; Chan, T. F.; Huang, H. L.; Lee, C. Y.; Tsai, S.; Wu, P. W.; Yang, Y. C.; Wang, T. N. \& Lee, C. H. Fructose-Rich Beverage Intake and Central Adiposity, Uric Acid, and Pediatric Insulin Resistance. $J$. Pediatr., 171:90-6 e1, 2016.

Liu, S.H.; Cai, F.Y. \& Chiang, M.T. Long-Term Feeding of Chitosan Ameliorates Glucose and Lipid Metabolism in a High-Fructose-DietImpaired Rat Model of Glucose Tolerance. Mar Drugs, 13(12):730213, 2015.

Lozano, I.; Van der Werf, R.; Bietiger, W.; Seyfritz, E.; Peronet, C.; Pinget, M.; Jeandidier, N.; Maillard, E.; Marchioni, E.; Sigrist, S. \& Dal, S. High-fructose and high-fat diet-induced disorders in rats: impact on diabetes risk, hepatic and vascular complications. Nutr. Metab. (Lond.), 13: 15, 2016.

Lustig, R.H. Fructose: metabolic, hedonic, and societal parallels with ethanol. J. Am. Diet Assoc., 110(9):1307-21, 2010.

Lustig, R.H. Fructose: it's "alcohol without the buzz". Adv. Nutr., 4(2):22635, 2013.

Lytle, K.A. \& Jump, D. B. Is Western Diet-Induced Nonalcoholic Steatohepatitis in Ldlr-/- Mice Reversible? Plos one, 11(1): e146942, 2016.

Machado, M. \& Cortez-Pinto, H. Non-alcoholic steatohepatitis and metabolic syndrome. Curr. Opin. Clin. Nutr. Metab. Care, 9(5):637-42, 2006.

Machado, M.V. \& Cortez-Pinto, H. Non-alcoholic fatty liver disease: what the clinician needs to know. World J. Gastroenterol., 20(36):12956-80, 2014.

Malhotra, N. \& Beaton, M. D. Management of non-alcoholic fatty liver disease in 2015. World J. Hepatol., 7(30):2962-7, 2015.

Mamikutty, N.; Thent, Z. C.; Sapri, S. R.; Sahruddin, N. N.; Mohd Yusof, M. R. \& Haji Suhaimi, F. The establishment of metabolic syndrome model by induction of fructose drinking water in male Wistar rats. Biomed. Res. Int., 2014:263897, 2014.

Marinho, T. S.; Kawasaki, A.; Bryntesson, M.; Souza-Mello, V.; Barbosada-Silva, S.; Aguila, M. B. \& Mandarim-de-Lacerda, C.A. Rosuvastatin limits the activation of hepatic stellate cells in diet-induced obese mice. Hepatol. Res., doi: 10.1111/hepr.12821) 2016 Epub ahead of print.

Maslak, E.; Buczek, E.; Szumny, A.; Szczepnski, W.; Franczyk-Zarow, M.; 
Kopec, A.; Chlopicki, S.; Leszczynska, T. \& Kostogrys, R.B. Individual CLA Isomers, c9t11 and t10c12, Prevent Excess Liver Glycogen Storage and Inhibit Lipogenic Genes Expression Induced by HighFructose Diet in Rats. Biomed. Res. Int., 2015:535982, 2015.

Matherly, S. C. \& Puri, P. Mechanisms of simple hepatic steatosis: not so simple after all. Clin. Liver Dis., 16(3):505-24, 2012.

Mishra, P. \& Younossi, Z. M. Abdominal ultrasound for diagnosis of nonalcoholic fatty liver disease (NAFLD). Am. J. Gastroenterol., 102 (12):2716-7, 2007.

Nguyen, T. A. \& Sanyal, A. J. Pathophysiology guided treatment of nonalcoholic steatohepatitis. J. Gastroenterol. Hepatol., 27 Suppl. 2: 58-64, 2012.

Osei-Hyiaman, D.; DePetrillo, M.; Pacher, P.; Liu, J.; Radaeva, S.; Batkai, S.; Harvey-White, J.; Mackie, K.; Offertaler, L.; Wang, L. \& Kunos, G. Endocannabinoid activation at hepatic CB1 receptors stimulates fatty acid synthesis and contributes to diet-induced obesity. J. Clin. Invest., 115(5):1298-305, 2005.

Pektas, M. B.; Sadi, G. \& Akar, F. Long-Term Dietary Fructose Causes Gender-Different Metabolic and Vascular Dysfunction in Rats: Modulatory Effects of Resveratrol. Cell Physiol. Biochem., 37(4):140720, 2015.

Petaja, E. M. \& Yki-Jarvinen, H. Definitions of Normal Liver Fat and the Association of Insulin Sensitivity with Acquired and Genetic NAFLDA Systematic Review. Int. J. Mol. Sci., 17(5), 2016.

Ramachandran, P.; Iredale, J.P. \& Fallowfield, J.A. Resolution of liver fibrosis: basic mechanisms and clinical relevance. Semin. Liver Dis., 35(2):119-31, 2015

Riquelme, A.; Arrese, M.; Soza, A.; Morales, A.; Baudrand, R.; Perez-Ayuso, R. M.; Gonzalez, R.; Alvarez, M.; Hernandez, V.; Garcia-Zattera, M. J.; Otarola, F.; Medina, B.; Rigotti, A.; Miquel, J. F.; Marshall, G. \& Nervi, F. Non-alcoholic fatty liver disease and its association with obesity, insulin resistance and increased serum levels of C-reactive protein in Hispanics. Liver Int., 29(1):82-8, 2009.

Rodrigues, A. H.; Moreira, C. C.; Mario, E. G.; de Souza Cordeiro, L. M.; Avelar, G. F.; Botion, L. M. \& Chaves, V. E. Differential modulation of cytosolic lipases activities in liver and adipose tissue by highcarbohydrate diets. Endocrine, 53 (2):423-32, 2016.

Schultz, A.; Barbosa-da-Silva, S.; Aguila, M. B. \& Mandarim-de-Lacerda, C.A. Differences and similarities in hepatic lipogenesis, gluconeogenesis and oxidative imbalance in mice fed diets rich in fructose or sucrose. Food Funct., 6(5):1684-91, 2015.

Schultz, A.; Neil, D.; Aguila, M. B. \& Mandarim-de-Lacerda, C. A. Hepatic adverse effects of fructose consumption independent of overweight/ obesity. Int. J. Mol. Sci., 14(11):21873-86, 2013.

Schwimmer, J. B.; Celedon, M. A.; Lavine, J. E.; Salem, R.; Campbell, N.; Schork, N. J.; Shiehmorteza, M.; Yokoo, T.; Chavez, A.; Middleton, M. S. \& Sirlin, C. B. Heritability of nonalcoholic fatty liver disease. Gastroenterology, 136(5):1585-92, 2009.

Sharma, N.; Li, L. \& Ecelbarger, C. M. Sex differences in renal and metabolic responses to a high-fructose diet in mice. Am. J. Physiol. Renal Physiol., 308(5):F400-10, 2015.

Singh, S.; Allen, A. M.; Wang, Z.; Prokop, L. J.; Murad, M. H. \& Loomba, R. Fibrosis progression in nonalcoholic fatty liver vs nonalcoholic steatohepatitis: a systematic review and meta-analysis of paired-biopsy studies. Clin. Gastroenterol. Hepatol., 13(4):643-54 e1-9; quiz e39-40, 2015.

Sodhi, K.; Puri, N.; Favero, G.; Stevens, S.; Meadows, C.; Abraham, N. G.; Rezzani, R.; Ansinelli, H.; Lebovics, E. \& Shapiro, J.I. Fructose Mediated Non-Alcoholic Fatty Liver Is Attenuated by HO-1-SIRT1 Module in Murine Hepatocytes and Mice Fed a High Fructose Diet. PLoS One, 10(6):e0128648, 2015.

Song, M.; Chen, T.; Prough, R.A.; Cave, M.C. \& McClain, C.J. Chronic Alcohol Consumption Causes Liver Injury in High-Fructose-Fed Male Mice Through Enhanced Hepatic Inflammatory Response. Alcohol Clin. Exp. Res., 40(3):518-28, 2016.

St Pierre, T. G.; House, M. J.; Bangma, S. J.; Pang, W.; Bathgate, A.; Gan,
E. K.; Ayonrinde, O. T.; Bhathal, P. S.; Clouston, A.; Olynyk, J. K. \& Adams, L. A. Stereological Analysis of Liver Biopsy Histology Sections as a Reference Standard for Validating Non-Invasive Liver Fat Fraction Measurements by MRI. PLoS One, 11(8):e0160789, 2016.

Stanhope, K. L. Sugar consumption, metabolic disease and obesity: The state of the controversy. Crit. Rev. Clin. Lab. Sci., 53(1):52-67, 2016.

Stanhope, K. L.; Medici, V.; Bremer, A.A.; Lee, V.; Lam, H. D.; Nunez, M. V.; Chen, G. X.; Keim, N. L. \& Havel, P. J. A dose-response study of consuming high-fructose corn syrup-sweetened beverages on lipid/ lipoprotein risk factors for cardiovascular disease in young adults. $\mathrm{Am}$. J. Clin. Nutr., 101(6):1144-54, 2015.

Stanhope, K. L.; Schwarz, J. M. \& Havel, P. J. Adverse metabolic effects of dietary fructose: results from the recent epidemiological, clinical, and mechanistic studies. Curr. Opin. Lipidol., 24(3):198-206, 2013.

Sumida, Y.; Nakajima, A. \& Itoh, Y. Limitations of liver biopsy and noninvasive diagnostic tests for the diagnosis of nonalcoholic fatty liver disease/nonalcoholic steatohepatitis. World J. Gastroenterol., 20(2):47585, 2014.

Sun, R.Q.; Wang, H.; Zeng, X. Y.; Chan, S. M.; Li, S. P.; Jo, E.; Leung, S. L.; Molero, J.C. \& Ye, J. M. IRE1 impairs insulin signaling transduction of fructose-fed mice via JNK independent of excess lipid. Biochim Biophys. Acta, 1852(1):156-65, 2015.

Tam, J.; Liu, J.; Mukhopadhyay, B.; Cinar, R.; Godlewski, G. \& Kunos, G. Endocannabinoids in liver disease. Hepatology, 53 (1):346-55, 2011.

Tanwar, S.; Trembling, P. M.; Guha, I. N.; Parkes, J.; Kaye, P.; Burt, A. D.; Ryder, S. D.; Aithal, G. P.; Day, C. P. \& Rosenberg, W. M. Validation of terminal peptide of procollagen III for the detection and assessment of nonalcoholic steatohepatitis in patients with nonalcoholic fatty liver disease. Hepatology, 57(1):103-11, 2013.

Tappy, L. \& Le, K. A. Metabolic effects of fructose and the worldwide increase in obesity. Physiol. Rev., 90(1):23-46, 2010.

Tappy, L. \& Le, K. A. Health Effects of Fructose and Fructose-Containing Caloric Sweeteners: Where Do We Stand 10 Years After the Initial Whistle Blowings? Curr. Diab. Rep., 15(8):627, 2015.

Tillman, E. J.; Morgan, D. A.; Rahmouni, K. \& Swoap, S. J. Three months of high-fructose feeding fails to induce excessive weight gain or leptin resistance in mice. PLoS One, 9(9):e107206, 2014.

Troeger, J. S.; Mederacke, I.; Gwak, G. Y.; Dapito, D. H.; Mu, X.; Hsu, C. C.; Pradere, J. P.; Friedman, R. A. \& Schwabe, R.F. Deactivation of hepatic stellate cells during liver fibrosis resolution in mice. Gastroenterology, 143(4):1073-83 e22, 2012.

Yoshioka, K.; Hashimoto, S. \& Kawabe, N. Measurement of liver stiffness as a non-invasive method for diagnosis of non-alcoholic fatty liver disease. Hepatol. Res., 45(2):142-51, 2015.

Zheng, R.; Liu, C.; Wang, C.; Zhou, B.; Liu, Y.; Pan, F.; Zhang, R. \& Zhu, Y. Natural Course of Metabolically Healthy Overweight/Obese Subjects and the Impact of Weight Change. Nutrients, 8(7), 2016.

\section{Dirección de correspondencia: \\ Pamela Carvallo Semler \\ Universidad Católica de Temuco \\ Manuel Montt 56 \\ Temuco \\ CHILE}

\section{E-mail:pcarvallosemler@yahoo.es}

Recibido : 17-02-2017

Aceptado: 28-03-2017 